\title{
Robust gene selection methods using weighting schemes for microarray data analysis
}

\author{
Suyeon Kang and Jongwoo Song ${ }^{*}$ (iD
}

\begin{abstract}
Background: A common task in microarray data analysis is to identify informative genes that are differentially expressed between two different states. Owing to the high-dimensional nature of microarray data, identification of significant genes has been essential in analyzing the data. However, the performances of many gene selection techniques are highly dependent on the experimental conditions, such as the presence of measurement error or a limited number of sample replicates.

Results: We have proposed new filter-based gene selection techniques, by applying a simple modification to significance analysis of microarrays (SAM). To prove the effectiveness of the proposed method, we considered a series of synthetic datasets with different noise levels and sample sizes along with two real datasets. The following findings were made. First, our proposed methods outperform conventional methods for all simulation set-ups. In particular, our methods are much better when the given data are noisy and sample size is small. They showed relatively robust performance regardless of noise level and sample size, whereas the performance of SAM became significantly worse as the noise level became high or sample size decreased. When sufficient sample replicates were available, SAM and our methods showed similar performance. Finally, our proposed methods are competitive with traditional methods in classification tasks for microarrays.

Conclusions: The results of simulation study and real data analysis have demonstrated that our proposed methods are effective for detecting significant genes and classification tasks, especially when the given data are noisy or have few sample replicates. By employing weighting schemes, we can obtain robust and reliable results for microarray data analysis.
\end{abstract}

Keywords: Microarray data, Gene selection method, Significance analysis of microarrays, Noisy data, Robustness, False discovery rate

\section{Background}

Microarray technologies allow us to measure the expression levels of thousands of genes simultaneously. Analysis on such high-throughput data is not new, but it is still useful for statistical testing, which is a crucial part of transcriptomic research. A common task in microarray data analysis is to detect genes that are differentially expressed between experimental conditions or biological phenotype. For example, this can involve a comparison of gene expression between treated and untreated samples, or normal and cancer tissue samples. Despite the rapid

\footnotetext{
* Correspondence: josong@ewha.ac.kr

Department of Statistics, Ewha Womans University, Seoul, South Korea
}

change of technology and the affordable cost for conducting whole-genome expression experiments, many past and recent studies still have relatively few sample replicates in each group, which makes it difficult to use typical statistical testing methods. These two problems, high dimensionality and small sample size problems, have triggered developments of feature selection in transcriptome data analysis [1-9]. These feature selection methods can be mainly classified into four categories depending on how they are combined with learning algorithms in classification tasks: filter, wrapper, embedded, and hybrid methods. For details and the corresponding examples of these methods, we refer the reader to several review papers [10-18]. As many researchers commented, filter 
methods have been dominant over the past decades due to its strong advantages, although they are the earliest in the literature $[11-13,15,16]$. They are preferred by biology and molecular domain experts as the results generated by feature ranking techniques are intuitive and easy to understand. Moreover, they are very efficient because they require short computation time. As they are independent of learning algorithms, they can give general solutions for any classifier [15]. They also have a better generalization property as the bias in the feature selection and that of the classifier are uncorrelated [19]. Inspired by its advantages, we focus on the filter method in this study.

One of the most widely used filter-based test methods is significance analysis of microarrays (SAM) [1]. It identifies genes with a statistically significant difference in expression between different groups by implementing gene-specific modified $t$-tests. In microarray experiments, some genes have small variance so their test statistics become large, even though the difference between the expression levels of two groups is small. SAM prevents those genes from being identified as statistically significant by adding a small positive constant to the denominator of the test statistic. This is a simple but powerful modification for detecting differentially expressed genes, considering the characteristics of microarray data. Since its establishment, the SAM program has been repeatedly updated. The latest version is 5.0 [20].

We also aim to develop methods for detecting significant genes based on a deeper understanding of microarray data. Even when researchers monitor an experimental process and control other factors that might have an influence on the experiment, biological or technical error can still arise in high-throughput experiments. For example, when one sample among a number of replicated samples gives an outlying result owing to a technical problem, variance of the gene expression becomes larger than expected and its test statistic becomes small. This is a major issue because it can lead to biologically informative genes failing to be identified as having a significant effect. Therefore, we here attempt to reduce this increase in variance for such cases by modifying the variance structure of SAM statistics, using two weighting schemes. It is also important to adjust the significance level of tests. Since we generally need to test thousands of genes simultaneously, the multiple testing problem arises. To resolve this problem, several methods have been suggested as replacements for the simple $p$-value; for example, we can use the family-wise error rate (FWER), false discovery rate (FDR) $[1,21]$, and positive false discovery rate (pFDR) [22]. Among them, FDR, which is the expected proportion of false positives among all significant tests, is a popular method to adjust the significance level. It can be computed by permutation of the original dataset. The test procedures we propose in this paper also use FDR, the same as SAM.
Once a list of significant genes is established by a gene selection method, researchers may carry out further experiments such as real-time polymerase chain reaction to determine whether these reference genes are biologically meaningful. However, many genes may not be tested owing to limitations of time and resources. For example, even if hundreds of genes are included in a list of reference genes for a user-defined significance cutoff, researchers may just select a few top-ranked genes among them for further analyses. Therefore, it is very important that the genes are properly ranked in terms of their significance, especially for top-ranked genes [23, 24]. As such, in this paper, we focus on improving test statistics for each gene and assessing how well each test method identifies significant genes.

For microarray data analysis, a comparison of the performance of gene selection methods is difficult because we generally do not know the "gold standard" reference genes in actual experiments. In other words, we do not know which genes are truly significant. This is a common problem encountered in transcriptome data analysis, so most studies have focused on comparing classification performances, which are determined by the combination of the feature selection and learning algorithm. As these results are clearly dependent on the performance of learning method, we cannot compare the effectiveness of feature selection techniques definitively [16]. Therefore, in this paper, we generate spike-in synthetic data that allow us to determine which genes are truly differentially expressed between two groups. For this, we suggest a data generation method based on the procedure proposed by Dembélé [25]. By performing such simulations, we can see how the performance changes depending on the characteristics of the dataset, such as sample size, the proportion of differentially expressed genes, and noise level. In this study, we focus on comparing performance according to noise level as our goal is to efficiently detect significant genes in a noisy dataset. To verify that our proposed methods can also compete with previous methods for actual microarray data, we use two sets of actual data that have a list of gold standard genes based on previous findings. All of these real datasets are publicly available and can be downloaded from a website [26] and R package [27]. In order to compare different gene selection methods, we also define two performance metrics that can be used when true differentially expressed genes are known.

This paper is organized as follows. In the next section, we review the algorithm of SAM and propose statistical tests for microarray data that are modified versions of SAM, named MSAM1 and MSAM2. In addition, we explain our synthetic data generation method and suggest two performance metrics. In the results section, we describe our simulation studies and real data analysis. We 
compare SAM, MSAM1, and MSAM2 using 14 types of simulated dataset, which have different noise levels and sample sizes, and two sets of real microarray data. We next discuss the difference between the three methods in detail, focusing on FDR estimation. Additionally, we give the results of classification analysis using some topranked genes selected by each method. In the last section, we summarize and conclude this paper.

\section{Methods}

In this section, we briefly review the SAM algorithm [1] and propose new modified versions of SAM, focusing on calculating the test statistic.

\section{SAM}

Let $x_{i j}$ and $y_{i j}$ be the expression levels of gene $i$ in the $j$ th replicate sample in states 1 and 2, respectively. For such a two-class case, the states of samples indicate different experimental conditions, such as control and treatment groups. Let $n_{1}$ and $n_{2}$ be the numbers of samples in these two groups, respectively. The SAM statistic proposed in [1] is defined as follows:

$$
d_{i}=\frac{\bar{x}_{i}-\bar{y}_{i}}{s_{i}+s_{0}}
$$

where $\bar{x}_{i}$ and $\bar{y}_{i}$ are the mean expression of the $i$ th gene for each group, $\bar{x}_{i}=\sum_{j=1}^{n_{1}} x_{i j} / n_{1}$ and $\bar{y}_{i}=\sum_{j=1}^{n_{2}} y_{i j} / n_{2}$. The gene-specific scatter $s_{i}$ is defined as:

$$
s_{i}=\sqrt{a\left\{\sum_{j=1}^{n_{1}}\left(x_{i j}-\bar{x}_{i}\right)^{2}+\sum_{j=1}^{n_{2}}\left(y_{i j}-\bar{y}_{i}\right)^{2}\right\}}
$$

where $a=\left(1 / n_{1}+1 / n_{2}\right) /\left(n_{1}+n_{2}-2\right)$ and $s_{0}$ is a small positive constant called the fudge factor, which is chosen to minimize the coefficient of variation of $d_{i}$. The computation of $s_{0}$ is explained in detail in [3].

Now let us consider the overall algorithm. The SAM algorithm proposed in [1] can be stated as follows:

1. Calculate test statistic $d_{i}$ using the original dataset.

2. Make a permuted dataset by fixing the gene expression data and shuffling the group labels under the $H_{0}$ where $H_{0}: \bar{x}_{i}-\bar{y}_{i}=0$ for all $i$.

3. Compute test statistics $d_{i}^{*}$ using the permuted data and order them according to their magnitudes as $d_{(1)}^{*} \leq d_{(2)}^{*} \leq \cdots \leq d_{(n)}^{*}$, where $n$ is the number of genes.

4. Repeat steps 2 and $3 B$ times and obtain $d_{(1)}^{*}(b) \leq d_{(2)}^{*}$ $(b) \leq \cdots \leq d_{(n)}^{*}(b)$ for $b=1,2, \ldots, B$, where $B$ denotes the total number of permutations.

5. Calculate the expected score $d_{(i)}^{E}=\sum_{b=1}^{B} d_{(i)}^{*}(b) / B$.
6. Sort the original statistic from step $1, d_{(1)} \leq d_{(2)} \leq$ $\cdots \leq d_{(n)}$.

7. For user-specific cutoff $\Delta$, genes that satisfy $\mid d_{(i)}-$ $d_{(i)}^{E} \mid>\Delta$ are declared significant. A gene is defined as being significantly induced if $d_{(i)}-d_{(i)}^{E}>\Delta$ and significantly suppressed if $d_{(i)}-d_{(i)}^{E}<-\Delta$.

8. Define $d_{(\text {up) }}$ as the smallest $d_{(i)}$ among significantly induced genes and $d_{\text {(down) }}$ as the largest $d_{(i)}$ among significantly suppressed genes.

9. The false discovery rate (FDR) is defined as the proportion of falsely significant genes among genes considered to be significant and can be estimated as follows:

$$
\widehat{\mathrm{FDR}}=\frac{\sum_{b=1}^{B} \#\left\{i: d_{(i)}(b) \geq d_{(\text {up })} \vee d_{(i)}(b) \leq d_{(\text {down })}\right\} / B}{\#\left\{i: d_{(i)} \geq d_{(\text {up })} \vee d_{(i)} \leq d_{(\text {down })}\right\}}
$$

The algorithm consists of two parts: computation of the test statistic and determination of the cutoff for a given $\Delta$. We will focus on the first of these parts and apply a simple modification to the computation of genespecific scatter $s_{i}$ to find a more robust test statistic. The numerator of the modified statistic and that of the original SAM statistic are the same. All of the procedures can be implemented using the samr package for Bioconductor in R. [20] described how to use the package and provided technical details of the SAM procedure.

\section{Modified SAM}

From one experiment [28], we observed several cases in which most of the results of gene expression are very close to each other, apart from one substantial outlier. As a result, the ranks of these genes from SAM are lower than expected. This prompted us to propose a new test method that has a different variance structure, leading to robustness on identifying informative genes in the presence of outliers. Throughout the paper, we use the term "outliers" to indicate "unusual observations".

Let us consider two cases with the following data: case 1: $(5,5,5,5,8.54)$ and case $2:(3,4,5,6,7)$. For these two cases, the variance is the same, inferring that they have the same spread. However, even though the levels of variance are equal, in fact, we cannot say that the data points are similarly distributed. We believe that case 1 is more reliable than case 2. Our goal, therefore, is to propose a test statistic that has a more significant result for case 1 than for case 2 . To minimize the effects of outliers among samples, we use the median instead of the mean and employ a weight function $w$ when computing the test statistic, resulting in a less weight on an outlier sample that is far from other samples. A modified $s_{i}, \tilde{s_{i}}$, is defined as follows: 
$\tilde{s_{i}}=\sqrt{\sum_{j=1}^{n_{1}} w\left(x_{i j}\right)\left(x_{i j}-\operatorname{median}_{j}\left(x_{i j}\right)\right)^{2}+\sum_{j=1}^{n_{2}} w\left(y_{i j}\right)\left(y_{i j}-\operatorname{median}_{j}\left(y_{i j}\right)\right)^{2}}$

Accordingly, our test statistic $\tilde{d_{i}}$ is defined as follows:

$$
\widetilde{d_{i}}=\frac{\bar{x}_{i}-\bar{y}_{i}}{\widetilde{s_{i}}+s_{0}}
$$

Methods modified by this approach might be particularly useful when detecting differentially expressed genes from noisy microarray data. The key idea is to reduce the impact of outliers when calculating the test statistic. We propose two different weight functions in this paper. The values of $\tilde{s_{i}}$ and $\tilde{d_{i}}$ would differ quite markedly depending on the used weight function.

\section{Modified SAM1 (Gaussian weighted SAM)}

The weight function used in Modified SAM1 (MSAM1) is based on the Gaussian kernel, which is a widely used weight that decreases smoothly to 0 with increasing distance from the center. It is defined as follows:

$$
w\left(x_{i j} ; \mu_{i}, \sigma\right)=\frac{1}{\sigma} \phi\left(\frac{x_{i j}-\mu_{i}}{\sigma}\right)
$$

where $\phi$ is the probability density function of a standard normal distribution, $\phi(x)=e^{-x^{2} / 2} / \sqrt{2 \pi}$. The mean $\mu_{i}$ is a gene-specific parameter such that $\mu_{i}=\operatorname{median}_{j}\left(x_{i j}\right)$ and standard deviation $\sigma$ is a data-dependent constant determined by the following procedure: first, $m$ is defined as follows. $m=\max \left(\left|x_{i j}-\operatorname{median}_{j}\left(x_{i j}\right)\right|, \mid y_{i j}-\right.$ $\left.\operatorname{median}_{j}\left(y_{i j}\right) \mid\right)$. It is calculated from given data. Second, $p$ is a user-defined value between 0 and 1 . Finally, given $m$ and $p$, we can find the value of $\sigma$ that satisfies the following equation:

$$
m=F^{-1}(1-p ; 0, \sigma)
$$

where $F$ is the cumulative distribution function of a normal distribution. Therefore, $m$ would approximately be the $100(1-p)$ th percentile point of a normal distribution with mean 0 and standard deviation $\sigma$. As can be seen from Fig. 1, smaller $p$ yields smaller $\sigma$. Therefore, smaller $p$ makes the weight applied to outlier samples smaller. On the other hand, as $p$ increases, the results of original and modified SAMs become similar because the weight on the outlier is very similar to the weight on the nonoutliers. In this research, we set $p=0.001$ since we found that this value is sufficiently small to reduce the effect of outliers.

For a better understanding of MSAM1, we here illustrate the weight function of MSAM1 and its application in detail. Let us consider Leukemia data [29]; for details of this data, see real data analysis section. The data consist of 38 samples (27 from ALL patients and 11 from AML patients) and 7129 genes. For simplicity and clarity, we randomly selected five samples for each sample type and applied SAM, MSAM1 with $p=0.01$ and MSAM1 with $p=0.001$. In order to compare weights given by each method, let us take one gene, M96326_rna1_at (Azurocidin). This gene would be a good example to clarify the difference between SAM and MSAM1 because it has an outlier sample. From Fig. 2, we can see that gene expressions in group 1 are

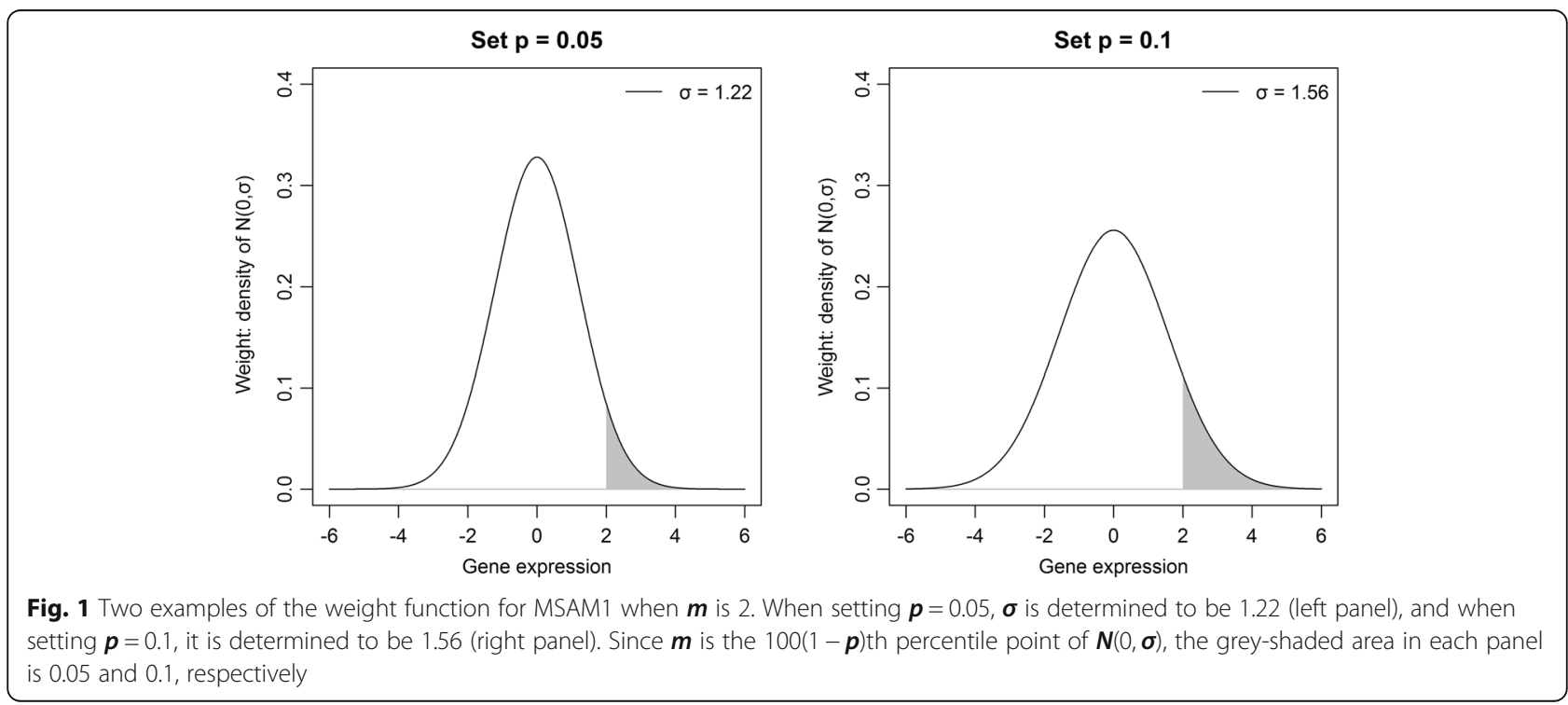




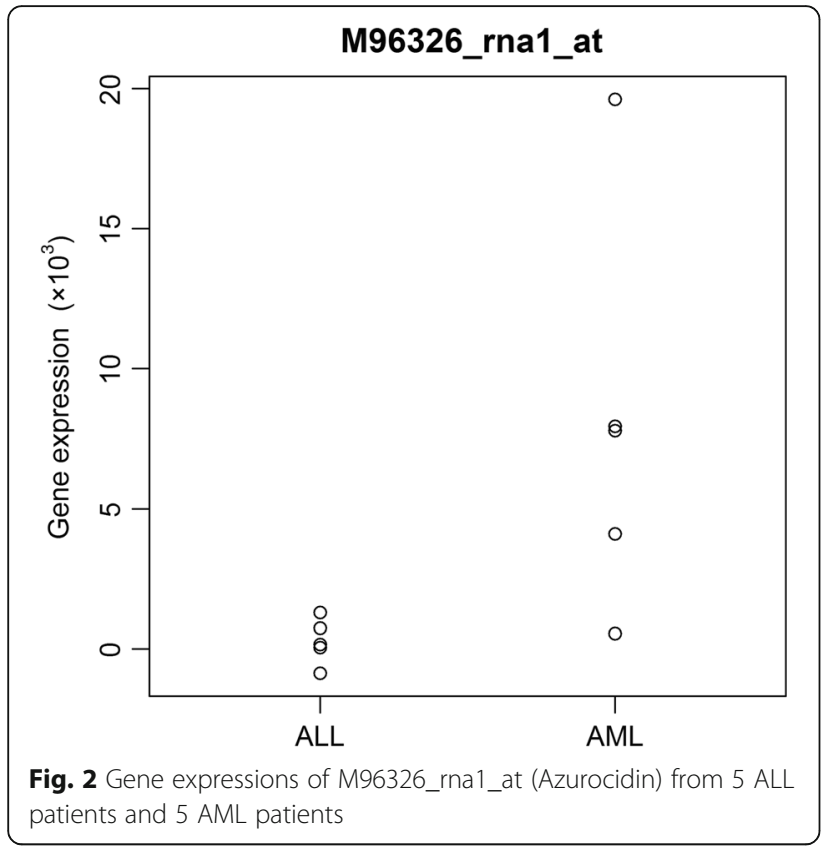

similar. On the other hand, one of five samples in group 2 is clearly far from others. Table 1 and Fig. 3 show its gene expressions and weights computed by SAM and MSAM1. In Fig. 3, the lengths of 5 red dashed lines indicate the weights on the 5 observations. As we stated above, we can also see that smaller $p$ makes the difference between weights applied to outlier and non-outlier samples greater.

\section{Modified SAM2 (inverse distance weighted SAM)}

This method uses Euclidean distance among the observations. The weight function used in Modified SAM2 (MSAM2) is defined as follows:

$$
w\left(x_{i j}\right)=\frac{1}{\sum_{k} d_{E}\left(x_{i j}, x_{i k}\right)}
$$

where $d_{E}\left(x_{i j}, x_{i k}\right)$ is the Euclidean distance between the $j$ th and $k$ th samples of gene $i$. The reason that we use this weight function can be explained by the following example. Let us assume that there are 10,000 genes $(i=$ $1,2, \ldots, 10000)$. Also, suppose there are 4 sample replicates (observations) in a group of the first gene $(i=$ $1)$ and their gene expressions are $x_{11}, x_{12}, x_{13}$ and $x_{14}$. Let $w_{j}$ be the weight on $j$ th observation for $j=1,2,3$ and 4. In this case, the weights on these observations are as follows.

$$
\begin{aligned}
& w_{1}=\left(\sum_{k=1}^{4} d_{E}\left(x_{11}, x_{1 k}\right)\right)^{-1}, \quad w_{2}=\left(\sum_{k=1}^{4} d_{E}\left(x_{13}, x_{1 k}\right)\right)^{-1}, \\
& w_{3}=\left(\sum_{k=1}^{4} d_{E}\left(x_{13}, x_{1 k}\right)\right)^{-1}, \quad w_{4}=\left(\sum_{k=1}^{4} d_{E}\left(x_{14}, x_{1 k}\right)\right)^{-1}
\end{aligned}
$$

If $x_{11}, x_{12}$ and $x_{13}$ are close to each other and $x_{14}$ is far from these 3 values, $w_{4}$ is much smaller than $w_{1}, w_{2}$ and $w_{3}$. Therefore, by using this weight function, we can give a smaller weight to an outlier. The further away an observation is from the others, the smaller weight is given.

\section{Synthetic data generation}

To run experiments, we need to generate synthetic gene expression data. These datasets should have characteristics similar to those of real microarray data to ensure that the results are reliable and valid. Two important characteristics of gene expression data, which are reported elsewhere $[25,30,31]$ and also considered in this study, are as follows:

1. Under similar biological conditions, the level of gene expression varies around an average value. In rare cases, technical problems would result in values far away from this average.

2. Genes at low levels of expression have a low signalto-noise ratio.

The 'technical problems' mentioned in the first of these points are one possible explanation for outliers observed in microarray data. Since our goal is to develop methods that detect differentially expressed genes well in a noisy dataset containing outliers, we consider not only a dataset with little noise, but also a noisy dataset with outliers. We ensure that outliers are present at higher probability in several of the datasets to provide a wider range of comparisons among the different test methods. Basically, we follow the microarray data gener-

\begin{tabular}{|c|c|c|c|c|c|c|c|c|c|c|}
\hline \multirow[b]{2}{*}{ Gene expressions $\left(\times 10^{3}\right)$} & \multicolumn{5}{|l|}{ ALL } & \multicolumn{5}{|l|}{ AML } \\
\hline & -0.86 & 0.05 & 0.16 & 0.74 & 1.30 & 0.55 & 4.11 & 7.79 & 7.96 & 19.60 \\
\hline SAM weights & 1.00 & 1.00 & 1.00 & 1.00 & 1.00 & 1.00 & 1.00 & 1.00 & 1.00 & 1.00 \\
\hline $\begin{array}{l}\text { MSAM1 weights }\left(\times 10^{-4}\right) \\
\text { for } p=0.01\end{array}$ & 0.37 & 0.37 & 0.37 & 0.37 & 0.37 & 0.30 & 0.35 & 0.37 & 0.37 & 0.20 \\
\hline $\begin{array}{l}\text { MSAM1 weights }\left(\times 10^{-4}\right) \\
\text { for } p=0.001\end{array}$ & 0.49 & 0.50 & 0.50 & 0.50 & 0.49 & 0.33 & 0.45 & 0.50 & 0.50 & 0.17 \\
\hline
\end{tabular}
ation model by Dembélé [25], which uses a beta

Table 1 Comparison of SAM and MSAM1 weights: an informative gene from leukemia data, M96326_rna1_at (Azurocidin) 

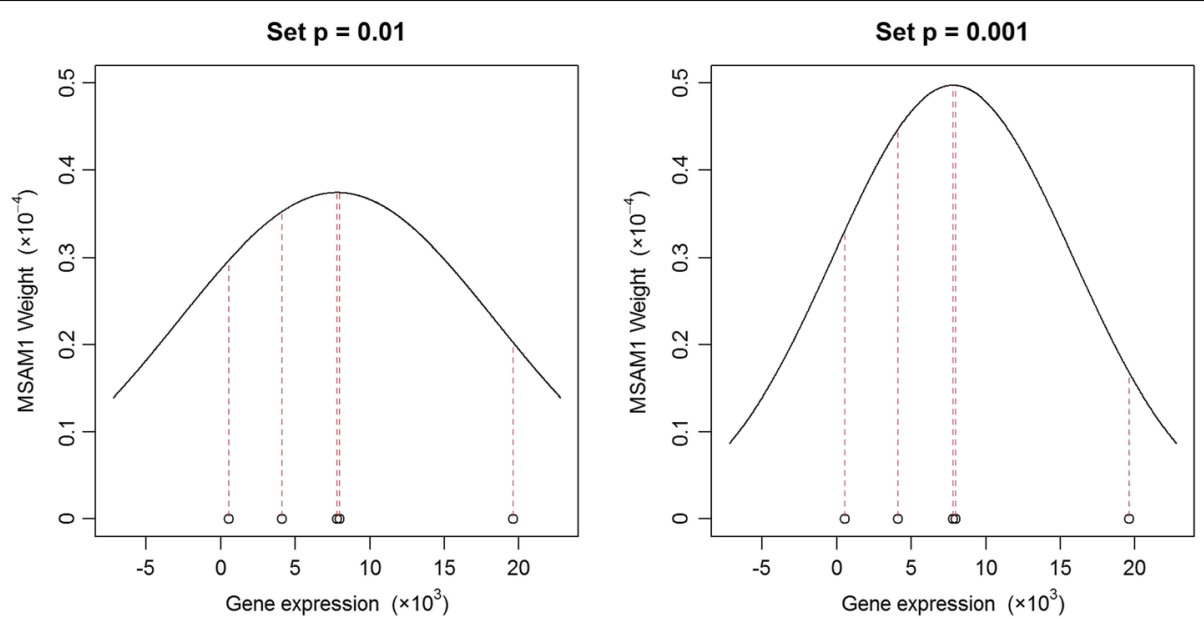

Fig. 3 The left panel illustrates the weights of MSAM1 when $p$ is 0.01 . The right panel is the case when $p$ is 0.001 . In each panel, 5 black circle points are gene expressions of M96326_rna1_at (Azurocidin) from 5 AML patients. The lengths of 5 red dashed lines indicate the weights on the 5 observations

distribution. In this article, we employ a beta and a normal distribution to generate data points, assuming that the levels of gene expression essentially follow such distributions. To allow outliers in generated data, we add a technical error term in our model; this term is mentioned in [25], but not used in their model. According to the noise level and distribution type, we consider four different simulation set-ups as follows: Scenario 1, noncontaminated beta; 2, contaminated beta; 3, noncontaminated normal; 4, contaminated normal. Therefore, data used in scenarios 1 and 3 have low noise level, and data used in scenarios 2 and 4 have high noise level. The step-by-step procedure for our data generation method is summarized as follows.

Step 1. Let $n$ be the number of genes and $n_{1}$ and $n_{2}$ be control and treatment sample sizes, respectively.

Step 2. Generate $z_{i}$ from a beta (normal) distribution for $i=1,2, \ldots, n$ and transform the values, $\bar{z}_{i}=l b+u b$ $\times z_{i}$.

Step 3. For each $\bar{z}_{i}$, generate $\left(n_{1}+n_{2}\right)$ values as follows: $z_{i j} \sim$ unif $\left(\left(1-\alpha_{i}\right) \bar{z}_{i},\left(1+\alpha_{i}\right) \bar{z}_{i}\right)$, where $\alpha_{i}=\lambda_{1} e^{-\lambda_{1} \bar{z}_{i}}$.

Step 4 . The final model is given by

$$
d_{i j}=z_{i j}+s_{i j}+n_{i j}+t_{i j}
$$

where the term $s_{i j}$ allows us to define differentially expressed genes. Their values are zero for the control group, $s_{i j} \sim N\left(\mu_{d e}, \sigma_{d e}^{2}\right)$ for genes with induced expression, and $s_{i j} \sim N\left(-\mu_{d e}, \sigma_{d e}^{2}\right)$ for genes with suppressed expression, where $\mu_{d e}=\mu_{d e}^{\min }+\operatorname{Exp}\left(\lambda_{2}\right) \cdot n_{i j}$ is an additive noise term, $n_{i j} \sim N\left(0, \sigma_{n}^{2}\right)$. The final term $t_{i j}$ is used to define outlying samples by allowing non-zero values for some genes. The undefined parameters for each step can be set by the users. The values we use in this paper are as follows: $\lambda_{1}=0.13, \lambda_{2}=2, \mu_{d e}^{\min }=0.5, \sigma_{d e}=0.5, \sigma_{n}=0.4$.
For these parameters, the influence of different parameter settings on the generated data is well explained elsewhere [25].

\section{Scenario 1: Beta with low noise level}

In this case, we generate data points from Beta(shape ${ }_{1}$, shape $_{2}$ ). shape 1 and shape $_{2}$ are two shape parameters of the beta distribution and we here set shape $_{1}=2$ and shape $_{2}=4$. We also set $l b=4, u b=14$. The values of $t_{i j}$ are zero for this case.

\section{Scenario 2: Beta with high noise level}

Here, we generate a noisier data than above data. The generation procedure is basically the same as the above case, except for allowing some non-zero $t_{i j}$. To make outlying samples, we contaminate the data by adding gaussian noise to some treatment samples: For genes with induced or suppressed expression,

$$
t_{i j} \sim N\left(0, \sigma_{\text {deo }}^{2}\right) \text { for } j=\left(n_{1}+n_{2}-n_{\text {deo }}+1\right), \ldots,\left(n_{1}+n_{2 .}\right)
$$

where $\sigma_{\text {deo }}$ is a non-zero constant and $n_{\text {deo }}$ is the number of outlying samples. We here set $\sigma_{\text {deo }}=1$ and $n_{\text {deo }}$ $=\left[0.2 \times n_{2}\right]$ where $[x]=m$ if $m \leq x<m+1$ for all integer $m$. For example, if there are five sample replicates in a treatment group, there can be one possible candidate as an outlier. Therefore, $\sigma_{\text {deo }}$ and $n_{\text {deo }}$ control the distribution and noise level of outlying samples. We believe that this set-up is reasonable because it does not destroy the original data structure while controlling the noise level of the data.

\section{Scenario 3: Normal with low noise level}

This scenario assumes that the levels of gene expression essentially follow a normal distribution, instead of a beta distribution. In this research, we use the normal 
distribution with mean 10 and standard deviation 1.5 for generated data points to be distributed between realistic bounds; the gene expression levels on a $\log 2$ scale after robust multichip analysis normalization usually vary between 0 and 20. We set $l b=0, u b=1$ in Step 2, which means that no transformation is applied.

\section{Scenario 4: Normal with high noise level}

To generate a noisier normal data, we use the same data generation procedure of Scenario 3, except for allowing some non-zero $t_{i j}$ in Step 4. The structure of $t_{i j}$ is the same as in Scenario 2.

\section{Performance metrics}

To compare the performance of several methods, we need several evaluation measures. Since we know which genes are differentially expressed in our simulated datasets, we can define two performance metrics as follows, measuring how well each method identifies these TRUE genes. Prior to define metrics, let $G_{\mathrm{up}}=\{i$ : gene $i$ the expression of which is truly significantly induced $\}$ and $G_{\text {down }}=\{i$ : gene $i$ the expression of which is truly significantly suppressed\}.

\section{Rank sum (RS)}

We define the rank sum (RS) of TRUE genes as follows:

$$
\mathrm{RS}=\sum_{i \in G_{u p} \cup G_{\text {down }}} \sum_{j: d_{i} d_{j}>0} \mathrm{I}\left(\left|d_{i}\right| \leq\left|d_{j}\right|\right)
$$

where $\mathrm{I}(\cdot)$ is an indicator function. The reason for determining the ranks of genes with high and low expression is that the SAM procedure uses such a method when detecting genes of the two groups. We use the absolute value of test statistics because test statistics of genes with suppressed expression have negative values. For RS, lower values indicate better performance.

\section{Top-ranked frequency (TRF)}

The top-ranked frequency (TRF) of TRUE genes is computed by

$$
\operatorname{TRF}(r)=\#\left\{i \in G_{\text {up }} \cup G_{\text {down }}: \sum_{j: d_{i} d_{j}>0} \mathrm{I}\left(\left|d_{i}\right| \leq\left|d_{j}\right|\right) \leq r\right\} .
$$

Here, $r$ denotes the rank cutoff and is set to be smaller than the number of observations in $G_{\text {up }}$ and $G_{\text {down }}$. For a given cutoff $r$, TRF computes the number of TRUE genes ranked within $r$. For TRF, higher values indicate better performance.

To understand the performance metrics better, let us consider the following case. We have 100 genes and 10 TRUE genes among them. Assume that we obtain a topranked gene list as shown in Table 2 by a gene selection
Table 2 An example list of top-ranked genes

\begin{tabular}{lll}
\hline Gene rank & Rank of true genes & True or false \\
\hline 1 & 1 & $\mathrm{~T}$ \\
2 & 2 & $\mathrm{~T}$ \\
3 & - & $\mathrm{F}$ \\
4 & 4 & $\mathrm{~T}$ \\
5 & 5 & $\mathrm{~T}$ \\
6 & 6 & $\mathrm{~T}$ \\
7 & - & $\mathrm{F}$ \\
8 & - & $\mathrm{F}$ \\
9 & 9 & $\mathrm{~T}$ \\
10 & 10 & $\mathrm{~T}$ \\
11 & 11 & $\mathrm{~T}$ \\
12 & - & $\mathrm{F}$ \\
13 & 13 & $\mathrm{~T}$ \\
14 & - & $\mathrm{F}$ \\
15 & 15 & $\mathrm{~T}$ \\
Rank sum & 76 & \\
\hline & & \\
\hline
\end{tabular}

method. Among the 15 genes in the table, five are false genes $\left(3^{\text {rd }}, 7^{\text {th }}, 8^{\text {th }}, 12^{\text {th }}\right.$, and $14^{\text {th }}$ genes in the table). In this case, $\operatorname{RS}=76, \operatorname{TRF}(5)=4$, and $\operatorname{TRF}(10)=7$.

\section{Results}

\section{Simulation studies}

In this section, we compare gene selection methods using synthetic datasets. We consider four scenarios described above. For each scenario, we consider 7 different combinations of $n_{1}$ and $n_{2}$ in order to take into account the affects of sample size and class imbalance on gene selection performance as follows: $\left(n_{1}, n_{2}\right)=(5,5),(5$, $10),(10,5),(10,10),(10,15),(15,10)$ and $(15,15)$. For all scenarios, we assume that there are $2 \%$ target genes (1\% up-regulated and 1\% down-regulated genes) among the total of 10,000 genes. For simplicity, let us assume that the first 100 genes are downregulated and last 100 genes are upregulated. Then, we can describe the structure of our simulation data as shown in Fig. 4. This example illustrates the structure of noisy data containing outliers. In this case, the last two samples are outlying samples among 10 treatment samples of 200 target genes. There are five different distributions of data points: A, B, C, D, and E. For 9800 nontarget genes, the distributions of the control and treatment samples are the same (A). The first 100 downregulated genes are generated from two distributions ( $\mathrm{B}$ and $\mathrm{C}$ ) and the last 100 upregulated genes are also generated from two distributions (D and E). Groups $\mathrm{C}$ and $\mathrm{E}$ indicate outlier samples. If there are no outliers in the dataset, B is equivalent to $C$ and $D$ is equivalent to $E$. The empirical density plot of each group is shown in Fig. 5. For 


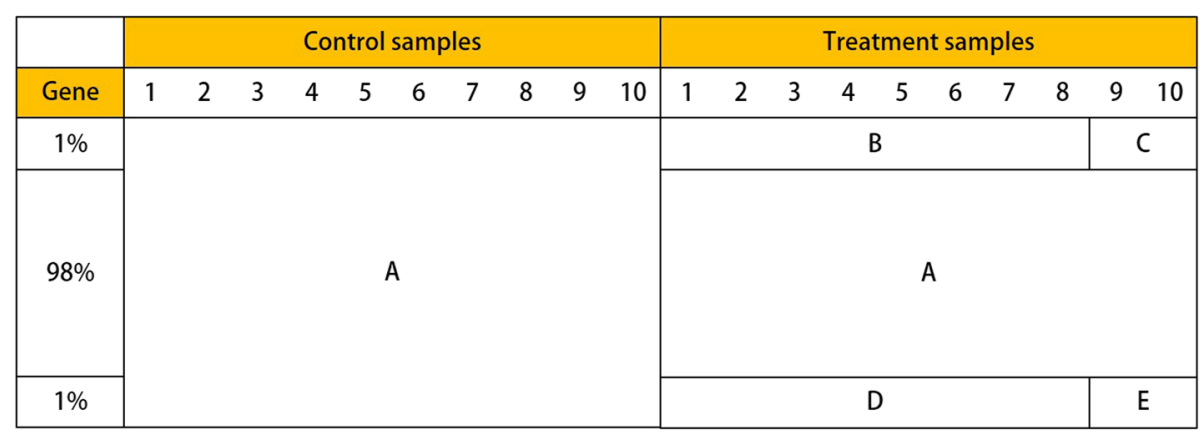

Fig. 4 An example of simulated data structure. Each row and each column of this data frame correspond to a gene and a replicate sample, respectively, so we have a $10,000 \times 20$ data matrix in this study. We assume that there are $2 \%$ target genes ( $1 \%$ up-regulated and $1 \%$ down-regulated genes) among the total of 10,000 genes, and ten replicates in each group. There are five different distributions of data points: $A, B, C, D$, and E; groups $C$ and $E$ indicate outlier samples

visualization, we use 5000 data points to ensure equivalent density of the points for each group (A, B, and C), that is, with a 1:1:1 ratio, not using the original ratio among the three groups.

We conduct simulation studies using synthetic data and compare the results using three metrics; two of them are RS and TRF, which were defined above, and the third is AUC. AUC is the area under a receiver operating characteristic (ROC) curve. Therefore, this value falls between 0 and 1 , and higher values indicate better performance. We consider five gene selection methods, named SAM, SAM-wilcoxon, SAM-tbor, MSAM1 and MSAM2. SAM-wilcoxon is the Wilcoxon version of SAM [20, 32]. SAM-tbor is basically the same with SAM, except for applying a simple trim-based outlier removing algorithm to data prior to running SAM. In this study, we remove the largest and smallest observations from each sample type. Figs. 6 and 7 display the average
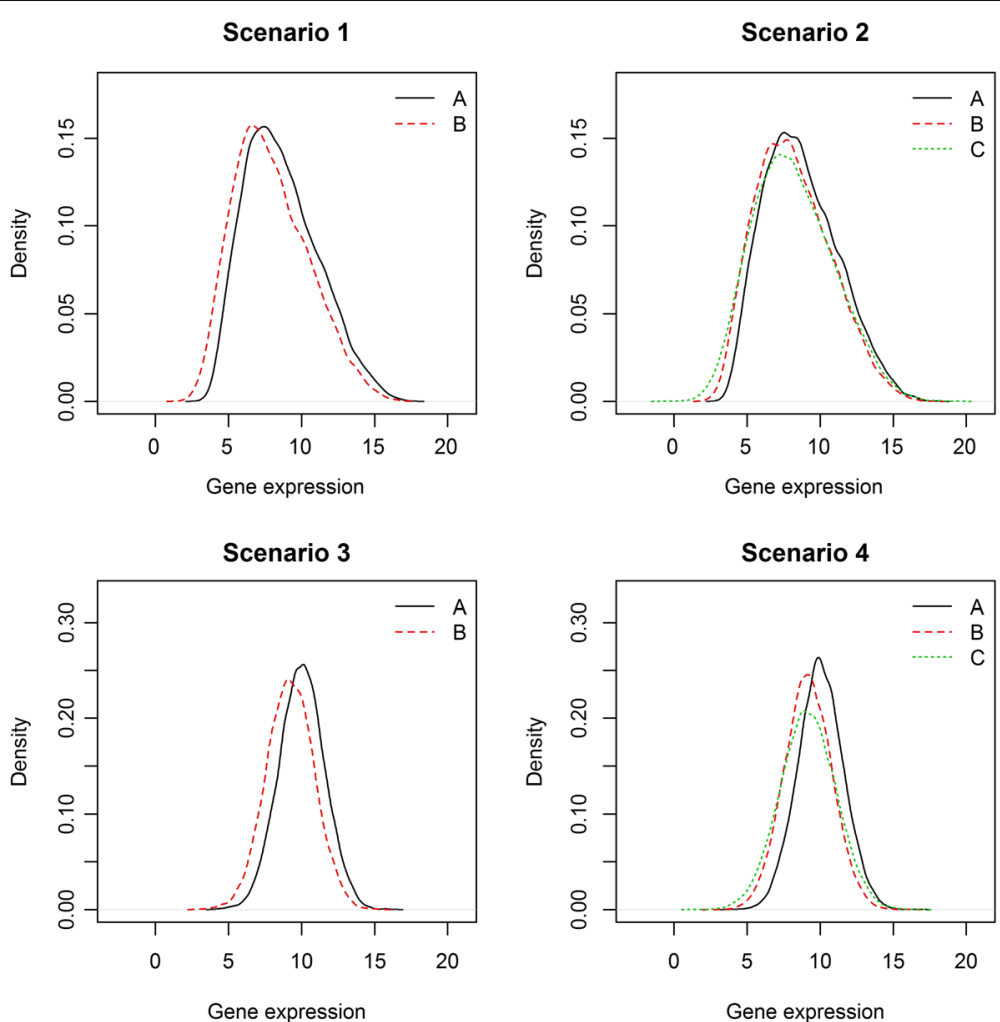

Fig. 5 Empirical density of data points for scenarios 1, 2, 3, and 4. The solid line (a) for each plot is the density of control samples for target genes (a). The red dashed line (b) and green dotted line (c) are the densities of treatment samples for target genes. There are no green dotted lines (c) in the top-left and top-right plots because there are no outliers in scenarios 1 and 3 


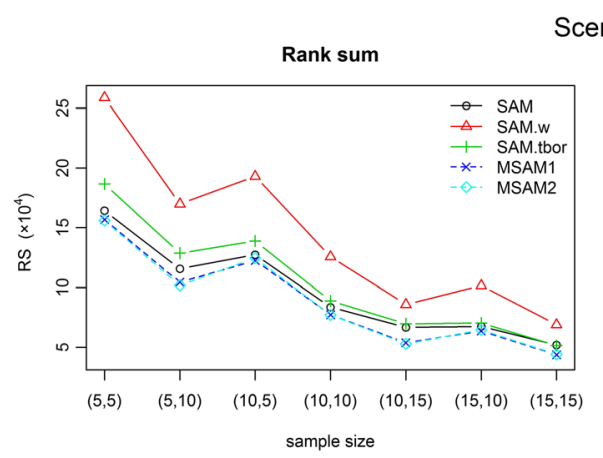

Scenario 1

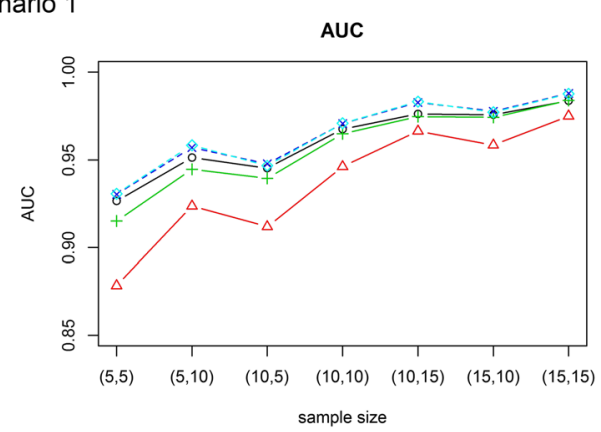

Top-ranked frequency (Top 100 genes)

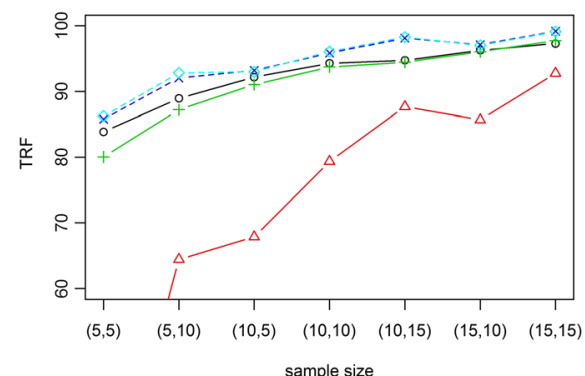

Top-ranked frequency (Top 200 genes)

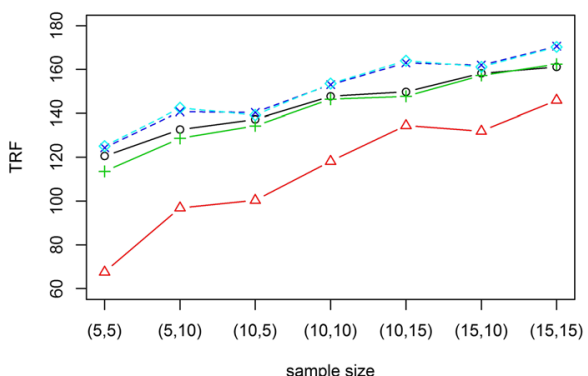

Fig. 6 Simulation results for Scenario 1. Three solid lines (black, red, and green) indicate the results of three versions of SAM. Two dashed lines (blue and cyan) indicate the results of two versions of modified SAM. For RS, lower values are better. For AUC and TRF, higher values are better

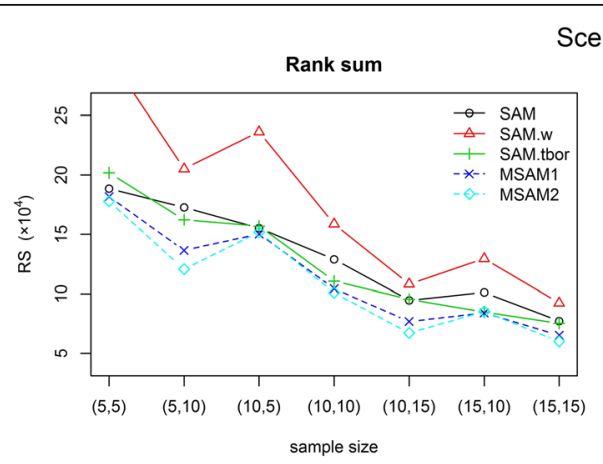

Scenario 2

Top-ranked frequency (Top 100 genes)
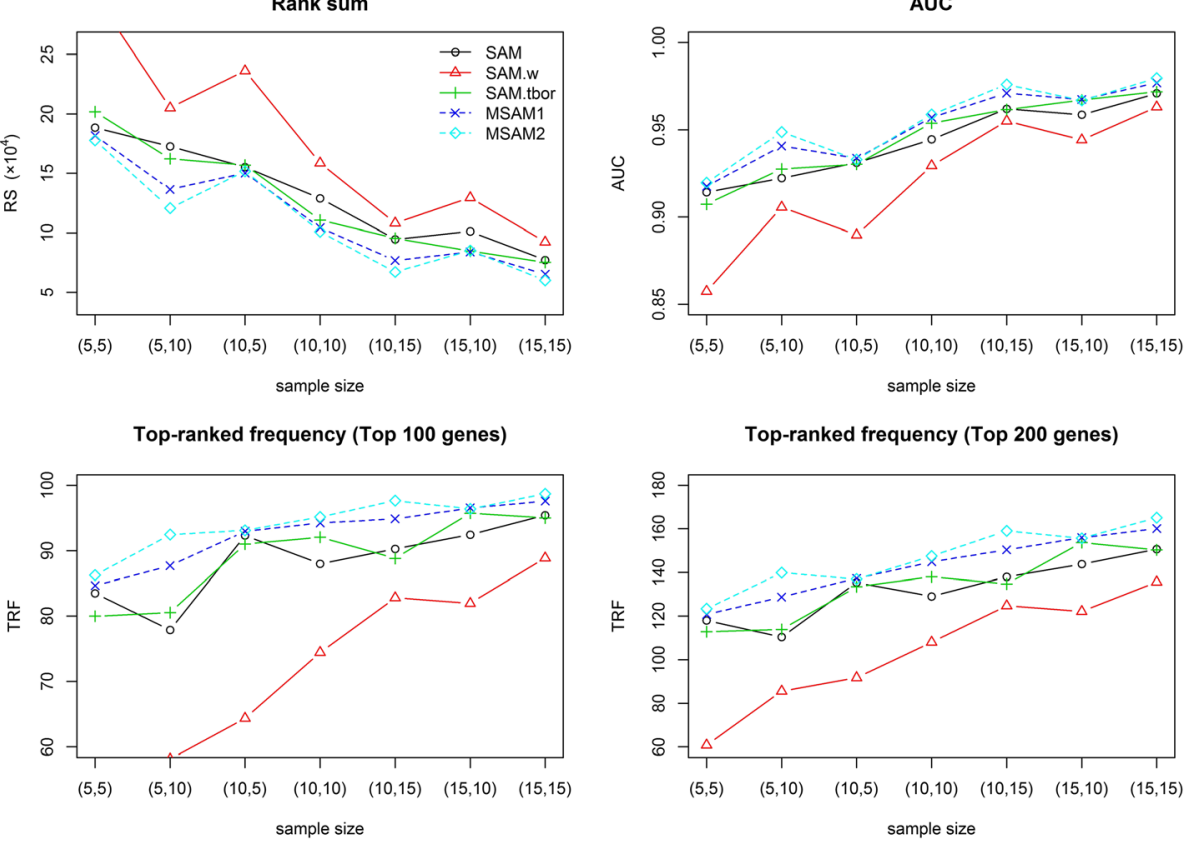

Top-ranked frequency (Top 200 genes)

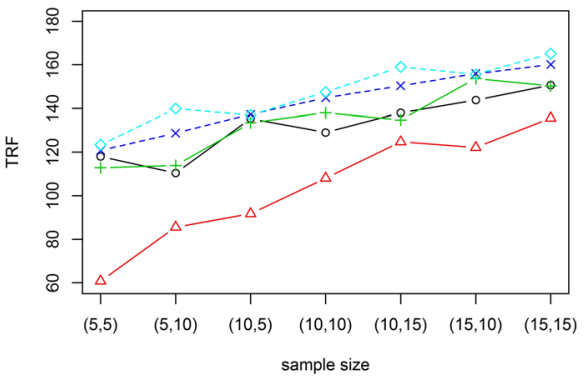

Fig. 7 Simulation results for Scenario 2 
performance of 100 simulations for each method on the three metrics. Table 3 shows numerical results of 4 cases. The best performance on each metric is shown in boldface. In scenario 1 , the original SAM always outperform SAM-wilcoxon and SAM-tbor. Although SAMtbor show better performance than SAM in some cases of scenario 2, its performance is worse than those of MSAMs. As can be seen from the figures and table, our proposed methods show better performance than three versions of SAM in all cases. In particular, modified SAMs are much better when given data is noisy (scenario 2, compared to scenario 1 ) and is a little better for less noisy cases. We can also see that our methods show more robust performance in all cases. When there is two outliers among ten samples, the number of target genes found by original SAM is reduced by $2-17 \%$, whereas that found by MSAMs is reduced by $1-8 \%$. In particular, when $n_{1}=5, n_{1}=10$ in scenario 2 , SAM fails to detect 90 genes among the 200 TRUE genes, whereas MSAM2 fails to detect only 60 genes on average. Simulation results of scenarios 3 and 4 are in Additional file 1. These results are very similar with those of scenarios 1 and 2; MSAMs always perform better than three versions of SAM.

\section{Real data analysis 1: Fusarium}

The Fusarium dataset contains 17,772 genes and nine samples: three each from control, dtri6, and dtri10 groups [28]. Robust multichip analysis algorithm is used for condensing the data for the following [33]: extraction of the intensity measure from the probe level data, background adjustments, and normalization. The post- processed dataset used in [28] are stored at PLEXdb (http://www.plexdb.org) (accession number: FG11) [26]. As this data was from gene mutation experiments, researchers provided a list of genes that are differentially expressed between control and treatment (dtri6, dtri10) groups. These genes are as follows: fgd159-500_at (conserved hypothetical protein), fgd159-520_at (trichothecene 15-O-acetyltransferase), fgd159-540_at (Tri6 trichothecene biosynthesis positive transcription factor), fgd159-550_at (TRI5_GIBZE - trichodiene synthase), fgd159-560_at, fgd159-600_at (putative trichothecene biosynthesis), fgd32160_at (trichothecene 3-O-acetyltransferase), fgd4-170_at (cytochrome P450 monooxygenase), fgd457-670_at (TRI15 - putative transcription factor), fg03534_s_at (trichothecene 15-O-acetyltransferase), fg03539_at (TRI9 - putative trichothecene biosynthesis gene), and fg03540_s_at (TRI11 - isotrichodermin C-15 hydroxylase).

In real data analysis sections, we only consider SAM, MSAM1, and MSAM2, all of which show good performance in simulation studies; we found that SAM-wilcoxon and SAM-tbor are worse than the original SAM in the previous section. Moreover, we cannot apply SAM-tbor to this data because this data has only three sample replicates in each group. Like this case, we can see that such a trim-based method is limited in its applications.

Tables 4 and 5 show the rank of 11 reference genes that are differentially expressed between the control group and the treatment groups (dtri6 and dtri10, respectively). The last row in each table indicates the rank sum of these 11 genes. As we can see, MSAM2 shows the best performance because the rank sum of this method is the smallest among those of the three gene

Table 3 Simulation results for 4 cases

\begin{tabular}{|c|c|c|c|c|c|c|c|c|}
\hline & \multicolumn{4}{|c|}{ Scenario $1, n_{1}=5, n_{2}=10$} & \multicolumn{4}{|c|}{ Scenario $1, n_{1}=10, n_{2}=10$} \\
\hline & RS & AUC & TRF & & RS & AUC & TRF & \\
\hline Rank cutoff & & & 100 & 200 & & & 100 & 200 \\
\hline SAM & 115,542 & 0.95 & 88.93 & 132.67 & 83,544 & 0.97 & 94.28 & 147.74 \\
\hline SAM-W & 169,865 & 0.92 & 64.43 & 96.84 & 125,513 & 0.95 & 79.37 & 118.18 \\
\hline SAM-tbor & 128,588 & 0.94 & 87.21 & 128.67 & 88,765 & 0.96 & 93.72 & 146.42 \\
\hline MSAM1 & 104,236 & 0.96 & 92.02 & 140.70 & 77,317 & 0.97 & 95.84 & 153.08 \\
\hline \multirow[t]{3}{*}{ MSAM2 } & 101,705 & 0.96 & 92.81 & 142.47 & 77,109 & 0.97 & 96.05 & 153.58 \\
\hline & \multicolumn{4}{|c|}{ Scenario $2, n_{1}=5, n_{2}=10$} & \multicolumn{4}{|c|}{ Scenario $2, n_{1}=10, n_{2}=10$} \\
\hline & RS & AUC & TRF & & RS & AUC & TRF & \\
\hline Rank cutoff & & & 100 & 200 & & & 100 & 200 \\
\hline SAM & 172,669 & 0.92 & 77.88 & 110.38 & 128,966 & 0.94 & 87.97 & 129.06 \\
\hline SAM-W & 205,161 & 0.91 & 58.14 & 85.55 & 158,618 & 0.93 & 74.44 & 108.06 \\
\hline SAM-tbor & 162,252 & 0.93 & 80.54 & 113.94 & 110,655 & 0.95 & 92.05 & 137.97 \\
\hline MSAM1 & 136,442 & 0.94 & 87.69 & 128.76 & 104,286 & 0.96 & 94.23 & 144.82 \\
\hline MSAM2 & 120,594 & 0.95 & 92.45 & 139.89 & 100,887 & 0.96 & 95.15 & 147.48 \\
\hline
\end{tabular}

Note: the best performance in each case is shown in bold type 
Table 4 Rank of genes of interest: control versus dtri6

\begin{tabular}{|c|c|c|c|c|c|c|c|}
\hline \multirow[b]{2}{*}{ Gene } & \multirow[b]{2}{*}{$\bar{x}_{i}-\bar{y}_{i}$} & \multicolumn{2}{|l|}{ SAM } & \multicolumn{2}{|l|}{ MSAM1 } & \multicolumn{2}{|c|}{ MSAM2 } \\
\hline & & $\widetilde{d_{i}}$ & rank & $\widetilde{d_{i}}$ & rank & $\tilde{d_{i}}$ & rank \\
\hline fgd457-670_at & -4.82 & -25.45 & 1 & -19.78 & 1 & -8.62 & 3 \\
\hline fgd159-550_at & -5.13 & -24.58 & 2 & -19.20 & 2 & -9.27 & 2 \\
\hline fgd159-600_at & -5.38 & -18.24 & 6 & -14.10 & 6 & -8.12 & 4 \\
\hline fg03534_s_at & -4.48 & -14.70 & 7 & -10.29 & 7 & -5.64 & 7 \\
\hline fg03540_s_at & -3.34 & -13.56 & 8 & -9.97 & 9 & -5.02 & 14 \\
\hline fgd321-60_at & -3.71 & -13.26 & 9 & -9.50 & 10 & -5.28 & 10 \\
\hline fg03539_at & -3.80 & -13.21 & 10 & -10.07 & 8 & -5.19 & 12 \\
\hline fgd159-500_at & -3.66 & -12.49 & 11 & -9.01 & 11 & -5.39 & 9 \\
\hline fgd159-520_at & -5.08 & -11.60 & 12 & -8.70 & 12 & -5.62 & 8 \\
\hline fgd159-540_at & -4.06 & -10.73 & 18 & -8.59 & 13 & -5.03 & 13 \\
\hline fgd4-170_at & -4.98 & -9.22 & 26 & -7.58 & 21 & -5.21 & 11 \\
\hline Rank sum & & & 110 & & 100 & & 93 \\
\hline
\end{tabular}

Note: the best performance in terms of rank sum is shown in bold type

selection methods. In particular, MSAMs improve the rank of the genes named fgd4-170_at and fgd159-500_at. For each of these genes, the result for one of their treatment samples is far from those for the other two samples. From the analysis, it can be asserted that our proposed methods efficiently identify the genes whose replicate samples contain an outlier, such as fgd4-170_at and fgd159-500_at.

\section{Real data analysis 2: Leukemia}

Leukemia is a cancer of the bone marrow, where blood cells are made. In leukemia, abnormal blood cells are produced in the bone marrow and crowd out other normal blood cells. Depending on the type of abnormal blood cells that are multiplying, leukemia can be classified as acute lymphocytic leukemia (ALL) or acute

Table 5 Rank of interest genes: control versus dtri10

\begin{tabular}{|c|c|c|c|c|c|c|c|}
\hline \multirow[b]{2}{*}{ Gene } & \multirow[b]{2}{*}{$\bar{x}_{i}-\bar{y}_{i}$} & \multicolumn{2}{|l|}{ SAM } & \multicolumn{2}{|c|}{ MSAM1 } & \multicolumn{2}{|l|}{ MSAM2 } \\
\hline & & $\overline{\tilde{d}_{i}}$ & rank & $\overline{\tilde{d}_{i}}$ & $\overline{\text { rank }}$ & $\overline{\tilde{d}_{i}}$ & rank \\
\hline fg03539_at & -6.66 & -22.18 & 1 & -17.27 & 1 & -10.46 & 2 \\
\hline fg03534_s_at & -4.00 & -11.24 & 4 & -8.42 & 4 & -5.68 & 4 \\
\hline fgd159-560_at & -2.76 & -9.74 & 5 & -8.01 & 5 & -5.22 & 6 \\
\hline fgd159-600_at & -3.28 & -8.77 & 8 & -6.81 & 8 & -4.92 & 7 \\
\hline fgd159-520_at & -4.17 & -8.12 & 9 & -6.31 & 10 & -4.65 & 8 \\
\hline fgd457-670_at & -3.35 & -6.44 & 16 & -5.05 & 18 & -3.83 & 12 \\
\hline fgd4-170_at & -3.53 & -5.73 & 21 & -4.76 & 19 & -3.68 & 13 \\
\hline fgd159-550_at & -3.22 & -4.89 & 26 & -4.23 & 22 & -3.42 & 16 \\
\hline fg03540_s_at & -2.35 & -4.84 & 27 & -3.88 & 27 & -2.90 & 23 \\
\hline fgd321-60_at & -1.62 & -3.07 & 60 & -2.44 & 64 & -1.88 & 38 \\
\hline fgd159-500_at & -1.73 & -2.88 & 78 & -2.37 & 73 & -1.90 & 37 \\
\hline Rank sum & & & 255 & & 251 & & 166 \\
\hline
\end{tabular}

myeloid leukemia (AML). Identifying the type of leukemia is very important because patients should receive different treatments according to the disease type. [29] studied a generic approach to cancer classification based on gene expression and provided a list of $50 \mathrm{sig}$ nificant genes for classifying ALL and AML. After this study, this dataset has been widely used in transcriptomic analysis, e.g., $[34,35]$. This data are available in the golubEsets library in Bioconductor [27]. The original data consist of 38 samples (27 from ALL patients and 11 from AML patients) and 7129 genes. We randomly selected five, seven, and ten samples for each sample type and repeated this experiment 100 times for averaging because biological experiments usually have a small number of samples owing to limitations of time and resources. It is thus important that a method shows good performance even if the sample size is small.

The simulation results are shown in Table 6. In this table, RS and TRF values of three gene selection methods, which were computed by using 50 genes that are considered informative in [29] over 100 trials. For each case, the best performance is shown in boldface in the table. As we can see, MSAM1 or MSAM2 performs better than SAM in terms of RS and TRF, regardless of rank cutoff values. The overall performance of SAM and MSAM1 are very similar, but MSAM1 always performs slightly better than SAM. In the point of view of sample size, MSAM2 outperform SAM and MSAM1 when the sample size is very small, e.g., 5, and MSAM1 performs better than SAM and MSAM2 when the sample size is moderate, e.g., 7 and 10. As the sample size increases, all of the three methods identify informative genes better.

\section{FDR comparison}

In this section, we discuss the FDR estimation procedures of SAM, MSAM1, and MSAM2. FDR is used in SAM procedure in order to deal with a multiple testing problem. The SAM interface in R, samr package [20], provides a significant gene list based on the FDR value that is estimated by its internal function. We also construct our own interface for MSAMs in R, based on the samr package, in order to allow for users to apply our proposed methods to their transcriptome research; see Additional file 2. Users start the procedure by setting their desired FDR value (for example, 0.2). We will call this value 'estimated FDR'. Based on the estimated FDR, our procedure calculates the value of corresponding $\Delta$ and identifies potentially significant genes. In real applications, we do not know TRUE FDR, so the estimated FDR is used as a substitute for TRUE FDR. If the estimated value is different from the true value, the number of genes that are detected using the estimated FDR is larger or smaller than the true number. Therefore, users may be interested in how well SAM and MSAMs 
Table 6 Rank sum and top-ranked frequency of informative genes in Leukemia data

\begin{tabular}{|c|c|c|c|c|c|c|c|c|c|}
\hline & \multicolumn{3}{|c|}{ \# picked samples: 5} & \multicolumn{3}{|c|}{ \# picked samples: 7} & \multicolumn{3}{|c|}{ \# picked samples: 10} \\
\hline & & & & \multicolumn{6}{|c|}{ Rank sum } \\
\hline & SAM & MSAM1 & MSAM2 & SAM & MSAM1 & MSAM2 & SAM & MSAM1 & MSAM2 \\
\hline & 22,287 & 21,585 & 15,815 & 11,924 & 11,790 & 13,286 & 5566 & 5534 & 11,256 \\
\hline & & & & \multicolumn{6}{|c|}{ Top-ranked frequency } \\
\hline$r$ & SAM & MSAM1 & MSAM2 & SAM & MSAM1 & MSAM2 & SAM & MSAM1 & MSAM2 \\
\hline 20 & 5.05 & 5.29 & 7.46 & 7.56 & 7.86 & 9.05 & 11.93 & 12.15 & 10.34 \\
\hline 40 & 7.74 & 8.24 & 12.72 & 12.55 & 12.95 & 14.48 & 19.11 & 20.05 & 16.70 \\
\hline 60 & 10.30 & 11.19 & 16.46 & 16.33 & 17.09 & 18.02 & 25.10 & 25.89 & 20.23 \\
\hline 80 & 12.76 & 13.54 & 19.02 & 19.78 & 20.52 & 20.63 & 29.30 & 30.27 & 22.71 \\
\hline 100 & 14.67 & 15.74 & 21.36 & 22.59 & 23.52 & 23.07 & 32.72 & 33.54 & 24.75 \\
\hline 120 & 16.72 & 17.75 & 23.43 & 24.76 & 25.74 & 25.45 & 35.65 & 36.22 & 26.94 \\
\hline 140 & 18.38 & 19.48 & 25.25 & 26.90 & 27.67 & 27.29 & 37.77 & 38.16 & 28.91 \\
\hline 160 & 20.03 & 20.94 & 26.79 & 28.67 & 29.74 & 28.92 & 39.60 & 39.92 & 30.48 \\
\hline
\end{tabular}

Note: the best performance for each rank cutoff is shown in bold type

procedures estimate TRUE FDR value. To this end, in this section, we evaluate SAM, MSAM1, and MSAM2, focusing on their FDR estimation performances.

Since we know the number of TRUE significant genes in our simulated datasets, we can compare the estimated FDR and TRUE FDR in simulation study. After 100 simulations, we draw a scatter plot of the TRUE FDR versus the estimated FDR by calculating the average values of the TRUE FDR for each estimated FDR. We next draw a smooth curve close to the scatter plot for scenarios 1 and 2 to find the estimation accuracy at various levels of FDR. In particular, the estimation accuracy at low FDR is important since researchers generally set FDR at a small value so as to avoid having a large proportion of falsely significant genes among the detected genes. For this reason, we only show the results when the estimated FDR is lower than 0.5. Figure 8 displays the results; see the top two plots. As we can see, SAM estimates the TRUE FDR very accurately and two modified SAMs slightly overestimate the TRUE FDR. In other words, our methods have conservative property in their FDR estimation. However, the conservative estimation of FDR may not cause serious problems for the analysis when we use FDR as an upper bound of a tolerable error [36].

For such an analysis, the more important thing is how many non-significant genes are included in the detected genes. Because the truths are known in the simulated data, we can calculate the number of falsely detected genes among the identified genes. With the same number of total positives, the method with the smallest number of false positives is the best [36]. Using the plotting method described above, a smooth curve of the number of false positive genes versus the total number of identified genes are drawn. Figure 8 shows the results From the figure, we can see that MSAM1 and MSAM2 gives smaller number of false positive genes than SAM across all noise level and the total number of identified genes. From the results, we can say that MSAMs are better than SAM because they includes the less number of false genes in the selected gene subset.

When we estimate FDR, we calculate both median FDR and mean FDR to determine which estimate more closely approximates the true value. Since the original samr interface provides the median FDR and 90th percentile FDR only, we modified its estimation function and obtained the median and mean values of FDR. As a result, we found that the median FDR was closer than the mean FDR to the TRUE FDR for all methods. This coincides with results published elsewhere [37], in which the median FDR was recommended as a criterion for gene selection methods when the estimated proportion of differentially expressed genes is greater than $1 \%$, regardless of the sample size. Based on these results, we use the median value instead of the mean value when estimating FDR.

\section{Classification analysis}

Once important genes are identified from thousands of genes, they can be used to predict two different experimental states or responses (for example, cancer and normal). Therefore, we also examine how well a few top genes selected by each method identify the true classes. We attach these results in Additional file 3. In this file, we introduce 4 datasets we used and explain the construction of classifiers, 6 gene selection methods, 3 performance metrics to be considered in this study. Our comments on the results are also included. As can be seen in the file, our proposed methods, MSAMs, show quite good performances in all cases. In this additional 
Scenario 1

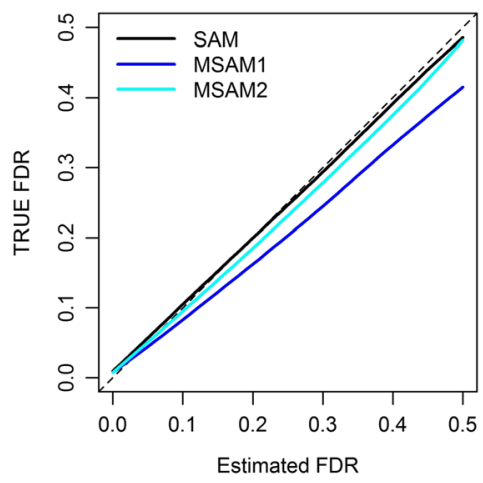

Scenario 1

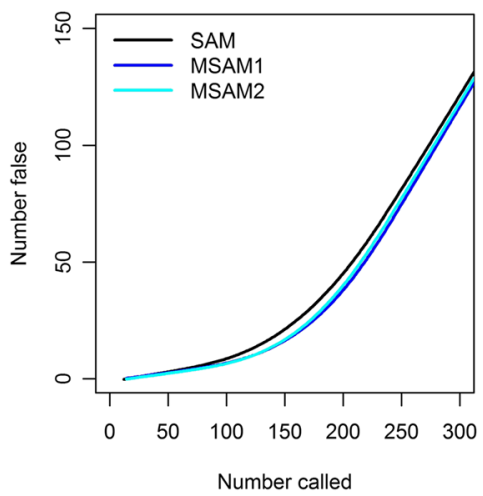

Scenario 2

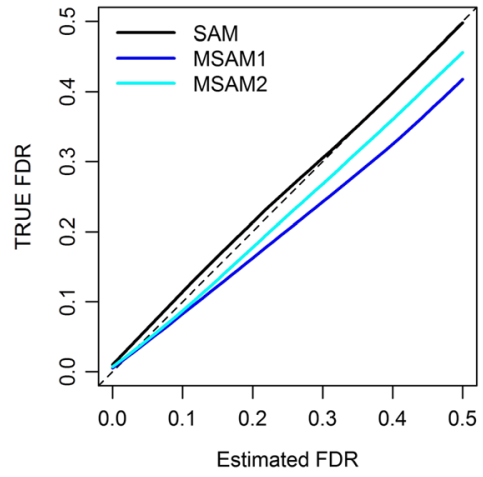

Scenario 2

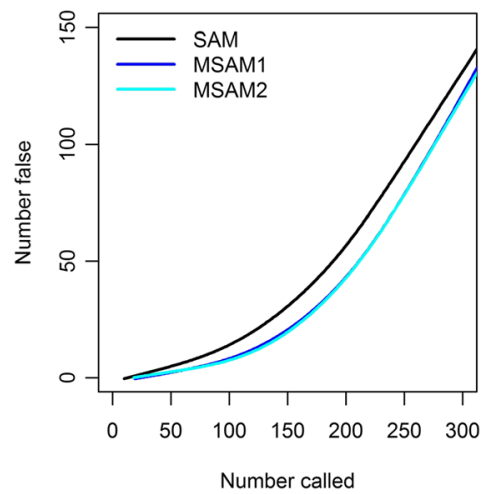

Fig. 8 The top two plots show TRUE FDR vs. estimated FDR and the bottom two plots show the number of falsely detected genes relative to the total number of detected genes for scenario 1 and 2. In each top plot, the solid lines indicate estimation curves of each method and the dashed line represents $\boldsymbol{Y}=\boldsymbol{X}$

section, we prove their competitiveness in classification tasks, not only in gene selection tasks.

\section{Discussion}

In transcriptome data analysis, most studies have been devoted to developing filter-based methods that are the simplest and fastest, and most computationally efficient. Hybrid methods, which are generally the combination of filter and wrapper methods, have recently gained popularity in the literature [13]. These methods consist of two steps: First, relevant features are selected by a filter method and the remaining features are eliminated. Second, a wrapper method verify these features and determine the final feature set that gives high classification accuracy [16]. In this point of view, filter methods have a lot of flexibility as they can be combined with not only any learning algorithm, but also any gene selection method, such as a wrapper method, resulting in a hybrid method. The performance of a hybrid method relies totally on the combination of filter and wrapper methods as well as the classifier [18]. We believe that accurate gene selection by filter methods clearly allow better classification accuracy. Therefore, our new filter-based methods will be useful not only in gene selection, but also constructing a good classifier in microarray applications.

Our experiments showed the efficiency of our methods; it was demonstrated that when the same number of genes were selected, our methods included the less number of false genes than the conventional method. Our results also strongly suggest that these newly proposed methods outperform the conventional method and show quite consistent performance, even with a high noise level and a small sample size. Given that noisy data and a small sample size are commonly encountered in microarray studies [30, 38-40], we believe that our methods will prove useful.

This research was based on the existing interface of SAM that was modified to apply our proposed methods. This modified version of the samr package is available in Additional file 2. We attempted to find a balance between flexibility and control in the usage of our methods by allowing users to set particular parameters and by minimizing the number of modifications to the original 
interface. Additional file 2 includes a detailed explanation of what we changed, but users can easily apply our methods to their own datasets without reading the manuscript in the first file, since we provide some simple and useful examples of detecting differentially expressed genes using our methods in Additional file 4. We also provide two real datasets and one simulated dataset used in this study (see Additional files 5, 6 and 7). All of the additional files are also available at author's homepage (http://home.ewha.ac.kr/ josong/MSAM/index.html).

\section{Conclusions}

We have proposed new test methods for identifying genes that are differentially expressed between two groups in microarray data and evaluated their performance using a series of simulated data and two real datasets. The results have demonstrated that our proposed methods identified target genes better than the original method, SAM, for both simulation studies and real data analysis. Using our weighting schemes, significant genes can be selected in a more robust manner by avoiding the overestimation of variance. In particular, these procedures are very effective when the given data are noisy or the sample size is limited. Therefore, they prevent technical or biological problems that can occur in biological experiments and data pre-processing from impeding accurate gene selection. We believe that our proposed methods can be applied to various datasets in other fields if they have characteristics similar to microarray data.

\section{Additional files}

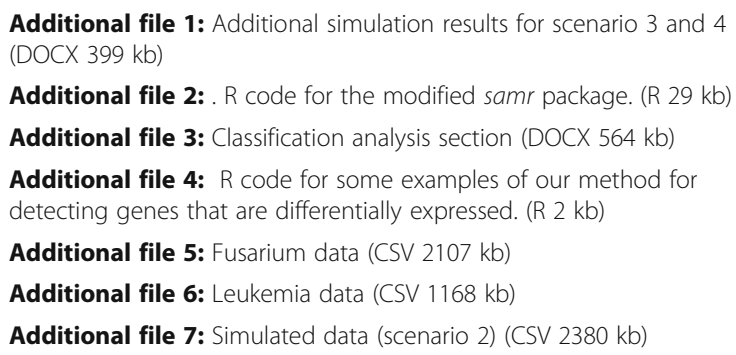

\section{Abbreviations}

ALL: acute lymphocytic leukemia; AML: acute myeloid leukemia; AUC: area under the curve; FDR: false discovery rate; MSAM1: modified SAM1; MSAM2: modified SAM2; ROC: receiver operating characteristic; RS: rank sum of true genes; SAM: significance analysis of microarrays; TRF: top-ranked frequency of true genes

\section{Acknowledgments}

The authors would like to thank the editor and three anonymous reviewers for their insightful comments that significantly improve this article.

\section{Funding}

This work was supported by the Ministry of Education of the Republic of Korea and the National Research Foundation of Korea (NRF-2017R1D1A1B03036078).
None of funding bodies played any role in the design or conclusions of this study.

\section{Availability of data and materials}

$\mathrm{R}$ code for implementing our proposed methods is in Additional files 2 and 4 of this article. The datasets supporting the conclusions of this article are included in Additional files 5, 6 and 7. All of these additional files are also available at http://home.ewha.ac.kr/ josong/MSAM/index.html.

\section{Authors' contributions}

All authors developed the approach, designed the study, wrote the computer code, analyzed the data, conducted the simulation studies and wrote the manuscript. All authors read and approved the final manuscript.

Ethics approval and consent to participate

Not applicable.

\section{Consent for publication}

Not applicable.

\section{Competing interests}

The authors declare that they have no competing interests.

\section{Publisher's Note}

Springer Nature remains neutral with regard to jurisdictional claims in published maps and institutional affiliations.

Received: 23 March 2017 Accepted: 27 August 2017

Published online: 02 September 2017

\section{References}

1. Tusher VG, Tibshirani R, Chu G. Significance analysis of microarrays applied to the ionizing radiation response. Proc Natl Acad Sci U S A. 2001;98(9): 5116-21.

2. Pavlidis P, Weston J, Cai J, Grundy WN. Gene functional classification from heterogeneous data. Proceedings of the fifth annual international conference on Computational biology. 2001:249-55.

3. Mak MW. Kung SY. A solution to the curse of dimensionality problem in pairwise scoring techniques. In neural information processing. Springer Berlin/Heidelberg. 2006:314-23.

4. Efron B. Microarrays, empirical Bayes and the two-groups model. Stat Sci. 2008;23(1):1-22.

5. Sharma A, Imoto S, Miyano S, Sharma V. Null space based feature selection method for gene expression data. Int J Mach Learn Cybern. 2012;3(4):269-76.

6. Sharma A, Imoto S, Miyano S. A between-class overlapping filter-based method for transcriptome data analysis. J Bioinforma Comput Biol. 2012;10(5):1-20.

7. Sharma A, Imoto S, Miyano SA. Top-r feature selection algorithm for microarray gene expression data. IEEE/ACM Trans Comput Biol Bioinform. 2012;9(3):754-64.

8. Ghalwash MF, Cao XH, Stojkovic I, Obradovic Z. Structured feature selection using coordinate descent optimization. BMC bioinformatics. 2016;17(1):158.

9. Sharbaf FV, Mosafer S, Moattar MHA. Hybrid gene selection approach for microarray data classification using cellular learning automata and ant colony optimization. Genomics. 2016;107(6):231-8.

10. Saeys $Y$, Inza I, Larranaga PA. Review of feature selection techniques in bioinformatics. Bioinformatics. 2007;23(19):2507-17.

11. Ahmad FK, Norwawi NM, Deris S. Othman NH. A review of feature selection techniques via gene expression profiles. In 2008 International Symposium on Information Technology

12. George G, Raj VC. Review on feature selection techniques and the impact of SVM for cancer classification using gene expression profile. arXiv preprint arXiv. 2011:1109-062.

13. Bolon-Canedo V, Sanchez-Marono N, Alonso-Betanzos A, Benitez JM, Herrera FA. Review of microarray datasets and applied feature selection methods. Inf Sci. 2014;282:111-35.

14. Tang J, Alelyani S, Liu H. Feature selection for classification: a review. Data Classification: Algorithms and Applications. 2014;37 
15. Ang JC, Mirzal A, Haron H, Hamed HNA. Supervised, unsupervised, and semi-supervised feature selection: a review on gene selection. IEEE/ACM Trans Comput Biol Bioinform. 2016;13(5):971-89.

16. Bolón-Canedo V, Sánchez-Maroño N, Alonso-Betanzos A. Feature selection for high-dimensional data. Prog. Artif Intell. 2016;5:65-75.

17. Mahajan S, Singh S. Review on feature selection approaches using gene expression data. Imp. J. Interdiscip. Res. 2016;2(3).

18. Aziz R, Verma CK, Srivastava N. Dimension reduction methods for microarray data: a review. AlMS. Bioengineering. 2017;4(1):179-97.

19. Ding $\mathrm{C}$, Peng H. minimum Redundancy feature selection from microarray gene expression data. J Bioinforma Comput Biol. 2005:3(2):185-205.

20. Chu G, Narasimhan B. Tibshirani R, and Tusher VG. SAM users guide and technical document: Stanford University Labs; 2005.

21. Benjamini $Y$, Hochberg $Y$. Controlling the false discovery rate: a practical and powerful approach to multiple testing. J R Stat Soc Ser B. 1995;57:289-300.

22. Storey JDA. Direct approach to false discovery rates. J R Stat Soc Ser B. 2002;64(3):474-98.

23. Mukherjee SN, Roberts SJ, Sykacek P, Gurr SJ. Gene ranking using bootstrapped p-values. SIGKDD Explor. 2003;5(2):16-22.

24. Boulesteix AL, Slawski M. Stability and aggregation of ranked gene lists. Brief Bioinform. 2009;10(5):556-68.

25. Dembélé DA. flexible microarray data simulation model. Microarrays. 2013;2(2):115-30.

26. Wise RP, Caldo RA, Hong L, Shen L, Cannon EK, Dickerson JA. BarleyBase/ PLEXdb: Plant Bioinformatics: Methods and Protocols. 2007:347?63.

27. http://www.bioconductor.org.

28. Seong KY, Pasquali M, Zhou X, Song J, Hilburn K, McCormick S, Dong $Y$, JR X, Kistler HC. Global gene regulation by fusarium transcription factors Tri6 and Tri10 reveals adaptations for toxin biosynthesis. Mol Microbiol. 2009;72(2):354-67.

29. Golub TR, Slonim DK, Tamayo P, Huard C, Gaasenbeek M, Mesirov JP, Coller H, Loh M, Downing JR, Caligiuri MA, Bloomfield CD, Lander ES. Molecular classification of cancer: class discovery and class prediction by gene expression monitoring. Science. 1999;286(5439):531?7.

30. Kooperberg CF, Aragaki AD, Strand A, Olson JM. Significance testing for small microarray experiments. Stat Med. 2005;24(15):2281-98.

31. Nykter $M$, Aho $T$, Ahdesmaki $M$, Ruusuvuori $P$, Lehmussola $A$, Yli-Harja O. Simulation of microarray data with realistic characteristics. BMC Bioinformatics. 2006;7(1):1.

32. Li J, Tibshirani R. Finding consistent patterns: a nonparametric approach for identifying differential expression in RNA-Seq data. Stat Methods Med Res. 2013;22(5):519-36.

33. Irizarry RA, Bolstad BM, Collin F, Cope LM, Hobbs B, Speed TP. Summaries of Affymetrix gene-Chip probe level data. Nucleic Acids Res. 2003;31(4):e15.

34. Pan W. A comparative review of statistical methods for discovering differentially expressed genes in replicated microarray experiments. Bioinformatics. 2002;18(4):546?54.

35. Zhang SA. Comprehensive evaluation of SAM, the SAM R-package and a simple modification to improve its performance. BMC Bioinformatics. 2007:8(1):230.

36. Xie Y, Pan W, Khodursky ABA. Note on using permutation-based false discovery rate estimates to compare different analysis methods for microarray data. Bioinformatics. 2005;21(23):4280-8.

37. Hirakawa A, Sato Y, Hamada D, Yoshimura IA. New test statistic based on shrunken sample variance for identifying differentially expressed genes in small microarray experiments. Bioinform Biol Insights. 2008;2:145-56.

38. Dougherty ER. Small sample issues for microarray?Based classification. Comp Funct Genomics. 2001;2(1):28-34

39. Marshall E. Getting the noise out of gene arrays. Science. 2004;306(5696): 630-1.

40. Cobb K. Microarrays: the search for meaning in a vast sea of data. Biomed. Comput Rev. 2006;2(4):16-23.

\section{Submit your next manuscript to BioMed Central and we will help you at every step:}

- We accept pre-submission inquiries

- Our selector tool helps you to find the most relevant journal

- We provide round the clock customer support

- Convenient online submission

- Thorough peer review

- Inclusion in PubMed and all major indexing services

- Maximum visibility for your research

Submit your manuscript at www.biomedcentral.com/submit
) Biomed Central 